\title{
AUGE Y OCASO DEL REGIONALISMO POST-LIBERAL: ENTRE LA CONVERGENCIA IDEOLÓGICA Y EL LIDERAZGO REGIONAL
}

\author{
María Victoria ALVAREZ1
}

RESUMEN: Una década después del surgimiento del regionalismo post-liberal, asistimos a fuertes movimientos en detrimento de su supervivencia, tanto en el caso de la UNASUR como de ALBA. ¿Qué factores explicaron la aparición de estos esquemas característicos del regionalismo post-liberal? ¿El debilitamiento de esos factores puede explicar el actual agotamiento de esta ola de regionalismo? Partimos del supuesto de que la convergencia ideológica y el liderazgo regional son facilitadores del regionalismo. Sin embargo, conjeturamos que la afinidad ideológica no puede por sí misma dar cuenta de los patrones variables en la cooperación regional. Especialmente en el periodo del regionalismo post-liberal, fue fundamental la voluntad de liderazgo de Brasil y Venezuela, de la mano de Lula da Silva y Hugo Chávez, y por consiguiente, tanto el surgimiento como el ocaso de ALBA y UNASUR pueden ser explicados predominantemente por los vaivenes del liderazgo regional.

PALABRAS CLAVE: Regionalismo post-liberal. UNASUR. ALBA. Liderazgo regional. Convergencia ideológica.

\section{THE RISE AND FALL OF POST-LIBERAL REGIONALISM: BETWEEN IDEOLOGICAL CONVERGENCE AND REGIONAL LEADERSHIP}

\footnotetext{
ABSTRACT: A decade after the rise of post-liberal regionalism, strong movements are emerging to the detriment of their survival, both in the case of UNASUR and ALBA. What

1 Universidad Nacional de Rosario, Facultad de Ciencia Política y Relaciones Internacionales, Rosario - Argentina. Doctora en Relaciones Internacionales. Profesora Adjunta. ORCID: https://orcid.org/0000-0002-2203-5082. maria.alvarez@fcpolit.unr.edu.ar.
} 
factors explained the emergence of these post-liberal regionalism schemes? Can the weakening of these factors explain the current exhaustion of this wave of regionalism? We assume that ideological convergence and regional leadership are facilitators of regionalism. However, we conjecture that ideological affinity by itself cannot account for variable patterns in regional cooperation. Especially in the period of post-liberal regionalism, Brazil's and Venezuela's will (with Presidents Lula da Silva and Hugo Chavez) to lead was central, and therefore, both the emergence and the decline of ALBA and UNASUR can be explained predominantly by the swings of regional leadership.

KEYWORDS: Post-liberal Regionalism. UNASUR. ALBA. Regional leadership. Ideological convergence.

\section{Introducción}

Luego de cinco décadas de subsecuentes olas de auge y decadencia del regionalismo en América Latina, en los primeros ańos del siglo XXI, gobiernos de izquierda recuperaron el espacio político y pusieron en debate los intereses, la racionalidad y los objetivos de la integración regional como se había aceptado hasta ese momento (SANAHUJA, 2012). Así, se dio el contexto propicio para el advenimiento de una nueva ola regionalista, caracterizada por la aparición de estructuras inéditas que se yuxtapusieron con esquemas preexistentes como el Mercado Común del Sur (MERCOSUR) y la Comunidad Andina de Naciones (CAN).

El regionalismo fue repensado en un diseño post-liberal (DA MOTTA; RÍOS, 2007; SANAHUJA, 2010, 2012), o post-hegemónico frente a Estados Unidos (RIGGIROZZI; TUSSIE, 2012), y que intentaba ir más allá del regionalismo abierto de la década anterior: en particular, una iniciativa innovadora de complementación y de cooperación materializada en la Alianza Bolivariana para los Pueblos de Nuestra América (ALBA), y un esquema de diálogo intersectorial y cooperación política, la Unión de Naciones Suramericanas (UNASUR). ${ }^{2}$ Por primera vez probablemente, los países latinoamericanos se permitieron diseñar esquemas regionales sin incorporar ni emular modelos foráneos. Pudieron reconsiderar la gobernanza regional en sus

\footnotetext{
2 A nivel regional se configuró la Comunidad de Estados Latinoamericanos y Caribeños (CELAC), como un instrumento de diálogo político y cooperación. La CELAC en los últimos tiempos ha perdido vigor y relevancia. Inclusive Brasil decidió suspender su membresía en enero de 2020. En este artículo nos centraremos en dos esquemas subregionales, UNASUR y ALBA.
} 
propios términos gracias a los nuevos márgenes de maniobra a nivel regional y subregional.

Una década después del surgimiento de estos esquemas con tono progresista, asistimos a fuertes movimientos en detrimento de su supervivencia. En un intento por sustituir a UNASUR emergió la iniciativa denominada Foro para el Progreso de América del Sur (PROSUR), de la mano de gobiernos de derecha y conservadores. ALBA, mientras tanto, se debate entre la inercia y el ocaso prematuro. ¿Qué factores explicaron la aparición del regionalismo post-liberal? ¿Es la disipación de esos factores lo que explica el actual agotamiento de esta ola de regionalismo?

En particular nos preguntamos ¿cuál es el papel que juega el liderazgo en la aparición y desaparición de los esquemas post-liberales? Y ¿cuál es el lugar que juega la ideología, más precisamente, la convergencia ideológica entre gobiernos de mismo signo político? En el artículo argumentamos que, más allá de la afinidad ideológica, fue la vocación de liderazgo de Lula da Silva y Hugo Chávez en el plano regional el rasgo distintivo que nos permite dar cuenta, entre otros factores, del surgimiento de UNASUR y ALBA. Asimismo, para explicar el ocaso de estos esquemas la falta de afinidad ideológica no es una variable tan significativa como el declive del liderazgo de Brasil y Venezuela.

Pese a la convergencia ideológica de los gobiernos durante el periodo estudiado, la cooperación en el seno de UNASUR se explica más por el liderazgo asertivo brasileño -especialmente durante el gobierno de Lula- que por la cohesión interna del organismo, al tiempo que la crisis interna de este país - a partir del gobierno de Dilma Rousseff - y su menor proyección externa significó un claro declive de Brasil como actor regional e internacional y de los esquemas de cooperación que había impulsado. En el caso de ALBA, la capacidad económica de Venezuela de cohesionar voluntades en torno a un proyecto común pesó más que la cercanía ideológica, y, por esta misma razón, la caída de los precios del petróleo y el declinante liderazgo venezolano no pudo evitar el debilitamiento del bloque, siendo que el peso del liderazgo de este país, especialmente durante la presidencia de Chávez, estaba atado a los altos precios internacionales del crudo.

Partimos del supuesto de que la convergencia ideológica y el liderazgo regional son facilitadores del regionalismo. Pero conjeturamos que la afinidad ideológica no puede por sí misma dar cuenta de los patrones variables en la cooperación regional. Especialmente en el periodo del regionalismo post-liberal, la convergencia ideológica estuvo acompañada de una clara voluntad y capacidad de dirigir por parte de algunos líderes regionales, como Brasil y Venezuela. En 
el mismo sentido, la concurrencia ideológica es un elemento insuficiente para mantener los consensos necesarios cuando el liderazgo declina.

La estrategia de investigación de este trabajo es el estudio de casos, en particular, el estudio de casos múltiples, con foco en ALBA y UNASUR, a través de técnicas cualitativas de recogida de información (revisión de fuentes de observación secundaria mediante bibliografía y documentos), y el uso de la técnica documental de análisis de datos. Reconocemos que nuestra hipótesis es de tipo correlacional, y no necesariamente intentamos verificar una conexión causal lineal entre las variables dependientes e independientes.

El artículo se organiza de la siguiente manera: luego de esta introducción realizamos algunas consideraciones de tipo conceptual y teórico para comprender la importancia de la convergencia ideológica en esquemas regionales deteniéndonos brevemente en algunos argumentos esbozados en la teorización sobre la integración europea y para el caso latinoamericano - y el rol de las potencias regionales en la gobernanza regional. A continuación, analizamos de manera separada la coincidencia ideológica y el liderazgo regional para comprender la aparición y el ocaso de UNASUR y ALBA. Finalizamos con algunas conclusiones y sugerencias para futuras investigaciones.

\section{Consideraciones teórico - conceptuales}

En las teorías sobre regionalismo, la ideología rara vez ha sido tenida en cuenta como variable independiente. En Europa, Haas, padre del neofuncionalismo, argumentó que las ideologías de los actores y la política desempeñan un papel secundario en la integración regional. En su opinión, no son las ideologías las que impulsan una integración más profunda, sino las necesidades funcionales (HAAS, 1958). Sin embargo, con posterioridad, Haas (1968) reconoció que prescindir de la variable de la ideología era erróneo, porque conducía a la exclusión de consideraciones políticas domésticas fundamentales para el análisis.

Desde la perspectiva del intergubernamentalismo liberal, por el contrario, Moravcsik (1998) observaba, a través de cinco períodos de estudios de integración europea, que los gobiernos a menudo han optado por instituciones supranacionales cuando sirven a sus propios intereses, independientemente de la ideología. Según Moravcsik, los factores geopolíticos, ideológicos e ideacionales han indudablemente influido en la integración europea, pero sólo marginalmente, y por lo tanto merecen menos atención.

En América Latina, los factores ideológicos juegan un rol preponderante (QUILICONI; RIVERA, 2019). Tal como afirman Rivarola Puntigliano y Briceńo 
Ruiz (2013) en su estudio sobre la resiliencia del regionalismo latinoamericano, no hay acciones políticas sin ideología. En la raíz de las acciones uno siempre encontrará ideologías y creencias que las guían. La ideología juega un papel clave en el regionalismo latinoamericano, que carece de instituciones independientes y resulta en gran medida del liderazgo de los presidentes (MALAMUD, 2015). La ideología es un factor esencial de integración, en contraposición a una visión del regionalismo basada principalmente en intereses materiales.

El éxito y el fracaso de la integración regional latinoamericana se explican, en parte, por la convergencia de las ideologías presidenciales en una organización determinada. A pesar de los obstáculos, la integración sigue siendo un tema fijo en la agenda oficial de los países latinoamericanos, e independientemente de las divisiones ideológicas, ningún gobierno rechaza la idea de integración (RIVAROLA PUNTIGLIANO; BRICEÑO RUIZ, 2013). De todas maneras, es el modelo de regionalismo el que cambia de un periodo a otro. La idea de cambio social y desarrollo que inspiró los esfuerzos de integración regional en América Latina durante las décadas de los 50 y 60 ha sido reemplazada por diferentes lógicas y visiones. En la actualidad, proyectos alternativos o antiliberales como ALBA y UNASUR están siendo desmantelados, después de una década conviviendo con esquemas resultantes del regionalismo abierto de los ańos 90.

$\mathrm{Al}$ interpretar el origen del regionalismo post-liberal, es posible distinguir al menos dos grupos de autores en la literatura especializada. Por un lado, algunos expertos destacan la importancia de la diplomacia presidencial y los componentes ideológicos como impulsores del regionalismo post-liberal. Sus raíces se hallan en el surgimiento de gobiernos de izquierda a principios de la década de 2000 (RIGGIROZZI; TUSSIE, 2012; LEGLER, 2013; PETERSEN; SCHULZ, 2018). Sin embargo, otro grupo de autores (MALAMUD; GARDINI, 2012; QUILICONI; SALGADO ESPINOZA, 2017) sostienen que aunque hubo una coincidencia en términos ideológicos de los gobiernos en el poder, el resultado fue incierto y la convergencia ideológica no logró profundizar el regionalismo sino que generó una proliferación de nuevas organizaciones.

En este trabajo argumentamos que la coincidencia ideológica es necesaria pero no suficiente para explicar los patrones del regionalismo de los últimos tiempos en América Latina: el liderazgo también juega un papel importante. La presencia de potencias regionales o potencias medias con una posición clara dentro de la región y el sistema internacional, y con ciertos atributos que permiten el ejercicio del liderazgo son fundamentales para explicar el surgimiento de formaciones regionales. Los Estados medios intentan reproducir su papel 
e intereses mediante el desarrollo de instituciones y formas de cooperación a nivel regional en apoyo de modelos específicos de desarrollo (RIGGIROZZI; TUSSIE, 2012).

La voluntad de los actores estatales es tan transcendental que es considerada por algunos especialistas como una de las condiciones sine qua non para que se desarrolle el regionalismo (MATTLI, 1999). El liderazgo es entendido como la capacidad y voluntad de uno o más actores de proveer monitoreo, implementación y coordinación colectiva o la presencia de un líder indisputado entre los países que buscan impulsar relaciones más cercanas (MALAMUD, 2010).

Ese líder sirve como punto focal en la coordinación de reglas, normas y políticas, y también puede convertirse en el paymaster, es decir, estar dispuesto a pagar una parte desproporcionada de los costos requeridos para el emprendimiento regional de manera de reducir las tensiones en torno a la distribución de estos (MATTLI, 1999). Tal como lo señalan los expertos, para que una potencia media sea aceptada como líder regional, debe proporcionar beneficios significativos a los países cuyo apoyo desea obtener.

La necesidad de un liderazgo se presenta entonces como un elemento central para crear (y sostener) una gobernanza regional. En la disciplina de las Relaciones Internacionales, es el estudio de la hegemonía el que posee una arraigada tradición. En particular, los enfoques realistas, los de la Economía Política Internacional, así como liberales y neoliberales, han dedicado grandes esfuerzos a explicar los ciclos hegemónicos y los momentos de transición hegemónica (HERRERA SANTANA, 2017). Sin embargo, la hegemonía constituye un concepto incómodo para aplicar sin más a países que no son considerados potencias, sino potencias medias o poderes emergentes, como Brasil o Venezuela. ${ }^{3}$

Más que a través de una estrategia hegemónica, la región puede construirse sobre la base de puntos de vista e intereses consensuados que, aunque avanzados y garantizados por el líder, reflejen la posición de los seguidores. Para algunos, esto implica una transacción entre el líder y los seguidores garantizando algún tipo de situación win-win en la que el líder puede utilizar la región como una plataforma para sus intereses, y los seguidores pueden beneficiarse de la

\footnotetext{
3 La utilización del término hegemonía en asociación con la política exterior de Brasil o Venezuela constituye una cuestión controvertida. En la raíz de esta afirmación se halla una aproximación a la hegemonía -especialmente visible en las concepciones clásicas sobre este tema- que prácticamente la equipara con la imposición y adhiere a un enfoque coercitivo del concepto, que considera la lucha por el poder como un juego de suma cero (KRASNER, 1976; KNORR, 1975; GILPIN, 1975).
} 
autonomía "negociada" y el acceso a recursos y mercados que de otro modo estarían restringidos (RIGGIROZZI; TUSSIE, 2012).

La idea de liderazgo, entonces, debe ser contextualizada y complementada, para nuestros propósitos, con alguna conceptualización de potencia regional. En este sentido, Nolte (2006) propone un concepto analítico de potencia regional al destacar los atributos de poder y las posibilidades de actuación de esta clase de Estados en un ámbito geográfico determinado.

De la conceptualización de Nolte (2006) de potencia regional, tomaremos en este trabajo sólo algunas de sus dimensiones. ${ }^{4}$ Entre ellas: el poder de exclusión (el líder influye en la delimitación geopolítica y construcción políticoideológica de la región, lo que puede marginar a ciertos países); la voluntad y rol/percepción (tiene la pretensión de un liderazgo en la región); el despliegue de recursos materiales (militares, económicos, demográficos), institucionales (políticos) e ideológicos para una proyección de poder en la región (recursos); el ejercicio efectivo de influencia en asuntos regionales (actividades y resultados); y la estructuración de la gobernanza regional (ejerce influencia en parte mediante estructuras de gobernanza regional).

Las dos variables estudiadas en este trabajo - convergencia ideológica y liderazgo regional - están vinculadas en el sentido de que la convergencia de las preferencias de los actores estatales para llevar adelante un determinado curso de acción constituye una condición para el inicio y la evolución de un proceso de cooperación o integración regional. La convergencia de preferencias entre los actores estatales puede venir dada por la afinidad ideológica, pero, en muchas ocasiones, requiere, asimismo, la acción de un líder regional.

\section{La convergencia ideológica en el origen del regionalismo post-liberal}

En términos generales, UNASUR y ALBA se caracterizaban por la convergencia de temas y áreas de acción en torno a tres "regresos": un "retorno de la agenda de desarrollo", un "retorno de la política" y un "retorno del Estado" (SERBIN, 2012, p.79). Más allá de las divergencias conceptuales, estratégicas e ideológicas y de los liderazgos en pugna que se reflejaban en estas dos propuestas, UNASUR era una iniciativa originalmente brasileña que le daba preferencia a las agendas política, social, de defensa y seguridad, en detrimento de la agenda comercial (SANAHUJA, 2012), mientras que ALBA, como esquema de cooperación Sur-Sur, destacaba por su oposición a Estados Unidos, la afini-

\footnotetext{
4 Para un análisis completo de estas dimensiones véase Alvarez (2020).
} 
dad ideológica de sus miembros y un discurso anti-hegemónico (GRATIUS; PUENTE, 2018).

UNASUR constituía una construcción regional diseñada por Brasil a partir de la anterior iniciativa de la Comunidad Sudamericana de Naciones (CSN), que se erigió sobre la base de intereses comunes en establecer una estructura institucional sudamericana en temas más allá del comercio, mientras buscaba al mismo tiempo una posición autónoma con relación a actores extrarregionales.

Los especialistas están divididos a la hora de evaluar el papel jugado por la convergencia ideológica de los gobiernos sudamericanos en la creación e institucionalización de UNASUR. Algunos colocan el énfasis en la existencia de dicha convergencia (SANAHUJA, 2012; QUILICONI; RIVERA, 2019) en virtud de un proceso de socialización fortalecido por la convergencia de discursos e ideologías. La narrativa unionista, restituida en Sudamérica como discurso "anti" o "post" neoliberal contribuyó a la generación de consensos y a la conformación de una cesta mínima de intereses comunes, que podían ser alcanzados a través de un marco regional (SANAHUJA, 2012). Otros expertos, no obstante, argumentan que la gran virtud del papel de Brasil fue justamente la de ejercer como árbitro, lograr consensos entre gobiernos con diferentes visiones político-ideológicas (RIGGIROZZI, 2012a; ALVES DO CARMO, 2012).

Consideramos que UNASUR era un proyecto más versátil que ALBA e incluso contradictorio, que abarcaba discursos disímiles de diferentes miembros. No había una sola identidad política e ideológica, aunque aparecía un nuevo espacio en oposición frente a la preeminencia estadounidense (RIGGIROZZI, 2012b). En tanto, ALBA reflejaba la visión socialista de Chávez. El componente ideológico buscaba como objetivo expandir el estado socialista de Venezuela a los países miembros (RIGGIROZZI, 2012b).

A través de UNASUR, especialmente durante el gobierno de Lula, la diplomacia brasileña trabajó con vistas a construir un consenso entre los países vecinos frente a situaciones dificultosas, y buscando siempre ocupar "un papel destacado" (GOMES SARAIVA, 2012, p.98). En especial, durante un corto tiempo, cuando la mayoría de los gobiernos sudamericanos eran progresistas, fue posible alinear parcialmente las agendas internacionales de los países de la región en función del proyecto de UNASUR y fue cuando mejores perspectivas se dieron en torno al bloque (FLEMES; WOJCZEWSKI, 2010).

Pero UNASUR incluía en su seno - al menos - dos modelos divergentes de inserción internacional, lo que condujo a "una lógica de mínimos denominadores comunes - que desembocó en principios y objetivos arraigados en altos niveles de abstracción - y a una arquitectura flexible y endeble" (COMINI; FRENKEL, 
2014, p.62). Esta lógica de consensos fue, en gran medida, un logro de la diplomacia brasileña.

Brasil orientó su comportamiento hacia la formación de consensos entre dos grupos de países que ostentaban modelos de inserción internacional disímiles. Por un lado, Argentina, Bolivia, Brasil, Ecuador, Paraguay y Venezuela; y, por otro lado, Chile, Colombia y Perú. ${ }^{5}$ Brasil utilizó a UNASUR como instrumento para facilitar las relaciones entre los países de la región, y actuó para encontrar "un punto intermedio entre las posiciones de estos dos grupos de países” (ALVES DO CARMO, 2012, p.314).

El esquema consiguió un exitoso impulso inicial que encaminó al organismo hacia un ciclo de crecimiento sostenido sus primeros años de andadura (COMINI; FRENKEL, 2014). UNASUR se sostuvo con una institucionalidad débil, priorizando la inclusión sobre la alineación ideológica, y tendió a evitar la cuestión crucial pero polémica de la integración económica (CUSACK, 2019).

Por su parte, ALBA se conformó en 2004, a partir de acuerdos entre la Venezuela de Chávez y la Cuba de Castro, como un esquema de asistencia y de cooperación Sur-Sur, con un fuerte contenido ideológico antiestadounidense, y que, con la inclusión del programa Petrocaribe - mecanismo mediante el cual Venezuela vendía petróleo en condiciones especiales a sus vecinos de la Cuenca del Caribe y Centroamérica -, reforzaba el contrapunto con el regionalismo abierto.

En abril de 2006, Bolivia decidió unirse a $\mathrm{ALBA}^{6}$ y junto con Cuba y Venezuela firmaron un Tratado de Cooperación de los Pueblos (TCP). ${ }^{7}$ En 2007, se unió Nicaragua al mando de Daniel Ortega, ${ }^{8}$ y en enero de 2008, la isla caribeña de Dominica. Chávez no logró reunir mucho más apoyo, excepto Ecuador - con el gobierno de Rafael Correa -, que adhirió en junio de 2009, y algunos países del Caribe insular que lo hicieron con posterioridad. ${ }^{?}$

\footnotetext{
5 Estos tres países, junto con México, conformaron la Alianza del Pacífico en 2011, que prioriza la apertura comercial y la integración de los países miembros en las cadenas globales de valor, contraponiéndose al protagonismo de una agenda política. Hoy, tras nueve años de vida, ha organizado quince cumbres presidenciales y despierta la atención de 59 observadores oficiales. Disponible en: https://alianzapacifico.net/. Acceso en: 12 ene. 2021.

6 Si bien lo hizo manteniendo su pertenencia en la CAN.

7 El acuerdo se centraba en temas sociales, con Cuba y Venezuela comprometidas en ayudar a Bolivia a reducir la pobreza y educar a los sectores más pobres de la población (DABÈNE, 2009).

8 A pesar de que este país había ratificado el Tratado de Libre Comercio entre República Dominicana, Centroamérica y Estados Unidos (DR-CAFTA, por sus siglas en inglés).

9 Fecha de incorporación al ALBA: Venezuela (2004), Cuba (2004), Bolivia (2006), Nicaragua (2007), Dominica (2008), Antigua y Barbuda, Ecuador y San Vicente y Las Granadinas (2009), Surinam y Santa Lucía (2012), Granada
} 
A partir del ingreso de Bolivia, ALBA se "multilateralizó" y se convirtió en una iniciativa que unió un heterogéneo grupo de países sudamericanos, centroamericanos y caribeños en torno al eje Cuba-Venezuela (GRATIUS; PUENTE, 2018, p.238). ALBA desarrolló una estrategia que significó una ruptura con las políticas neoliberales de la región de los años $90^{10}$, y comenzó a describirse como un bloque antiimperialista, lo que constituyó un hito en la etapa posthegemónica del regionalismo sudamericano (RIGGIROZZI; TUSSIE, 2012). Sobre la base de los principios de intercambio equitativo (frente al libre comercio), el beneficio mutuo (frente a la competencia), la cooperación, la justicia social, la soberanía nacional y el desarrollo sostenible, el ALBA era "un rechazo directo del neoliberalismo y manifiestamente anticapitalista” (RABY, 2011, p.169).

Venezuela pudo expandir la iniciativa de ALBA en el Caribe anglófono a través de Petrocaribe, proporcionando una especie de antecámara para que los caribeños aumentasen su contacto con los miembros latinoamericanos, a menudo como una plataforma hacia la membresía plena (CUSACK, 2019). Aquellos países que aceptaban la ayuda venezolana acababan por adoptar también su agenda referente al regionalismo, tal como lo manifiesta la parcialmente solapada membresía de ALBA y Petrocaribe. Caracas, dirigida por Chávez, comprendió que para que una potencia media sea aceptada como líder regional debía proporcionar beneficios significativos a los países más pequeños cuyo apoyo anhelaba. La estrategia funcionaba porque la posibilidad de obtener petróleo en condiciones especiales -especialmente en un contexto internacional de precios altos - y préstamos y ayudas sin ninguna condicionalidad - era una oferta que algunos países de la región simplemente no podían rechazar.

\section{El liderazgo regional en el origen del regionalismo post-liberal}

En los primeros años del siglo XXI, en América del Sur emergieron dos liderazgos destacados con capacidades diferenciadas, Venezuela y Brasil, de la mano de Chávez y Lula. Coincidimos con Quiliconi y Salgado Espinoza (2017) cuando sostienen que las iniciativas regionales en Sudamérica proliferaron en un modo à la carte en el cual las instituciones post-liberales fueron creadas para encarar temáticas relacionadas con los objetivos políticos y estratégicos de los líderes regionales. Tanto Brasil como Venezuela se posicionaron como líderes regionales, al poseer la mayoría de las dimensiones de un líder regional

y San Cristóbal y Nieves (2014). Honduras ingresó como miembro en 2008 bajo la presidencia de Manuel Zelaya, pero se retiró en 2009 luego de la destitución de éste.

${ }^{10}$ Con excepción de Cuba, que nunca formó parte de la ola neoliberal de los años 90. 
según Nolte (2006), y buscaron organizar a los países de la región en torno a sus proyectos. Sin embargo, en lugar de que la rivalidad entre los dos países fuese alimentada, las condiciones políticas permitieron que los proyectos de integración convergieran (ALVES DO CARMO, 2012).

UNASUR era una creación brasileña diseñada para promover el papel de Brasil, tanto a nivel regional como mundial (MALAMUD; GARDINI, 2012). $\mathrm{Y}$ aunque se ha dicho que "la UNASUR en sí misma puede ser vista como una iniciativa de Brasil y Venezuela" (FLEMES, WOJCZEWSKI, 2010, p.10), y que "Chávez contribuyó a forjar a UNASUR" (DABÈNE, 2009, p.220), estas observaciones no contradicen el hecho de que Brasil fue el líder más preponderante por el impulso otorgado a la CSN, y posteriormente a su sucesor directo, UNASUR, mientras que Venezuela se destacó por iniciativas como el ALBA y la "petro-cooperación" (LORENZINI; CEPPI, 2013).

Si bien es en la década de los 90 cuando Brasil orienta su política exterior hacia la región y promueve una iniciativa como el MERCOSUR, en la postGuerra Fría el regionalismo pasó a tener un lugar protagónico en la agenda de su política externa. El dispositivo central para avanzar en su proyecto de liderazgo fue "la creación de acuerdos regionales" (BURGES, 2009, p.11). Sin embargo, debemos advertir que el papel de Brasil en Sudamérica es tema de debate (MALAMUD, 2011; MOURÓN; ONUKI, 2015; CAETANO; LÓPEZ BURIAN; LUJÁN, 2019). Mientras un grupo de académicos atestigua el desarrollo del liderazgo regional de Brasil, otros cuestionan sus capacidades para construir dicho liderazgo. A estos dos grupos podríamos agregar un tercero, conformado por autores que sugieren que Brasil poseía las capacidades para ejercer el liderazgo sudamericano, pero que éste era objetado y por lo tanto no pudo consolidarse (MOURÓN; ONUKI, 2015).

En el año 2000, el presidente Cardoso organizó una primera reunión con los gobernantes de la región, con vistas a la formación de una comunidad sudamericana. Esta iniciativa condujo a la creación de la CSN en 2004, la cual, posteriormente, evolucionó hacia la UNASUR con un sesgo cooperativo fuerte, una agenda ampliada, y un carácter intergubernamental y de baja institucionalidad en términos prácticos. Brasil se alzaba como poder emergente y la política exterior liderada por Lula da Silva ponía a Sudamérica como área prioritaria para la inserción global del país (BURGES, 2009).

Si utilizamos el marco analítico de Nolte (2006), Brasil cumplía - en cierta medida - con la mayoría de los requisitos para ser una potencia regional (ALVAREZ, 2020). Ya en el segundo mandato de Cardoso, la idea de Sudamérica como una región única fue desarrollada como una base para sostener el status 
de Brasil como potencia regional/global. Esta tendencia fue reforzada bajo el gobierno de Lula da Silva, cuando la búsqueda de Brasil por el liderazgo regional en Sudamérica se intensificó (QUILICONI; SALGADO ESPINOZA, 2017). Esa delimitación geopolítica y construcción político-ideológica de la región incluía naturalmente a todos los países sudamericanos, pero implicaba diseñar un espacio geopolítico con poder de exclusión, al prescindir de todos aquellos al norte de la frontera norte de Colombia, en particular, México, pero también para ganar autonomía frente a Estados Unidos.

La realidad es que la creación de UNASUR le permitió a Brasil poner en movimiento otras dos premisas básicas del liderazgo de una potencia regional: el ejercicio efectivo de una importante influencia en asuntos regionales y la construcción de estructuras de gobernanza regional. Brasil amplió su esfera de influencia desde MERCOSUR a UNASUR e incluso a la CELAC, tomando al regionalismo como un paso para la construcción de su liderazgo regional (QUILICONI; SALGADO ESPINOZA, 2017).

$\mathrm{Si}$ bien los posibles seguidores han presentado recelos y han existido tensiones, en ningún momento habría existido un "rechazo frontal" al liderazgo brasileño como tal (MOURÓN; ONUKI, 2015, p.13). En todo caso, la intención no era buscar un liderazgo brasileńo de Sudamérica a través de la imposición, sino propiciar un ordenamiento beneficioso que impulsara los intereses, aspiraciones y estrategias brasileños en la región.

ALBA, por su parte, comenzó siendo una asociación entre Caracas y La Habana, que sumó gobiernos de países pequeños, como hemos notado. Este esquema rápidamente resultó ser un instrumento para la construcción de alianzas para Chávez, buscando maneras de consolidar el liderazgo en América Latina (DABĖNE, 2009). Venezuela poseía varios de los atributos de una potencia regional, en particular, ostentaba los recursos materiales - relativos, eso sí, al estado del ciclo internacional de los precios internacionales del petróleo - e ideológicos para una proyección de poder en la región (ALVAREZ, 2020).

Venezuela contó desde 2003 con un caudal significativo de recursos económicos que le permitió financiar su estrategia internacional de manera coyuntural. ${ }^{11}$ Además de diseñar el ALBA como contrapoder a Washington en la región, creó el bloque de Petrocaribe en 2005, inicialmente con 14 socios y

\footnotetext{
11 La diplomacia petrolera ha sido una constante en la política exterior venezolana en diferentes periodos históricos, con gobiernos autoritarios, liberales, de centroizquierda y neoliberales - los últimos tres durante la vigencia del puntofijismo.
} 
que llegó a contar con 18 naciones de Centroamérica y el Caribe en sus filas ${ }^{12}$ (LÓPEZ CANELLAS; VILANI, 2014). El formato de Petrocaribe era casi idéntico al que regía la compra de petróleo en condiciones de pago preferenciales entre Venezuela y Cuba. ${ }^{13}$

Su capacidad financiera dotó a Venezuela de capacidades económicas que le permitieron desarrollar a través del ALBA un "soft balancing" frente a Estados Unidos (BRICEÑO RUIZ, 2014; GRATIUS; PUENTE, 2018). El ALBA surgió como una forma de contrarrestar el objetivo estadounidense de crear el Área de Libre Comercio de las Américas (ALCA), y luego propuso un modelo de integración que rechazaba el modelo de los Tratados de Libre Comercio que Washington deseaba expandir en la región. Venezuela ejerció fuerte influencia en la delimitación geopolítica y la construcción político-ideológica para marginar a Estados Unidos, liderando el proceso de oposición a la iniciativa del ALCA.

Inclusive, para algunos, la exclusión implicaba también a Brasil. En esta línea, ALBA habría nacido "como un mecanismo para equilibrar la creciente influencia de Brasil en América del Sur, ya sea de forma unilateral o a través del liderazgo que ejerce en el MERCOSUR” (BRICEÑO RUIZ, 2012, p.143-145). En realidad, el bloque bolivariano intentaba impulsar una suerte de equilibrio frente a Brasil, no necesariamente de competencia (BRICEÑO RUIZ, 2012). Caracas veía a UNASUR como una oportunidad de llevar adelante sus estrategias energéticas hacia Sudamérica y promover el proyecto bolivariano del Presidente Chávez de integración regional (SANAHUJA, 2012).

$\mathrm{Al}$ interior de UNASUR, ALBA era una fuerza relevante y las propuestas de Chávez, que suavizadas, encontraban su camino en un proceso de toma de decisiones sudamericano más amplio. Especialmente, Brasil era quien "regionalizaba" y "sudamericanizaba" las propuestas venezolanas (SANAHUJA, 2012, p.49). Brasil, mostrando "liderazgo cooperativo", era capaz de incorporar los intereses de otros países de Sudamérica en UNASUR, aun cuando respondía al diseño geopolítico de ese país (SANAHUJA, 2016, p.44).

En síntesis, los recursos provenientes de la renta petrolera y el uso de una retórica ideologizada y antiestadounidense proveyeron a Venezuela con un conjunto de seguidores entre países pequeños de Centroamérica y el Caribe. En el Cono Sur, su influencia se sintió en estructuras regionales lideradas por

\footnotetext{
${ }^{12}$ Los 18 países eran: Antigua y Barbuda, Bahamas, Belice, Cuba, Dominica, El Salvador, Granada, Guyana, Haití, Honduras, Jamaica, Nicaragua, República Dominicana, San Cristóbal y Nieves, San Vicente y las Granadinas, Santa Lucía, Surinam, Venezuela. Guatemala se retiró en noviembre de 2013.

${ }_{13}$ El gobierno venezolano concedía un precio preferencial y fijo de U\$S 27 por barril pagado de la siguiente manera: el $50 \%$ de la factura a los 90 días con dos años de gracia y un plazo de 25 años. Venezuela asumía los gastos de flete y seguros por los barriles vendidos a Cuba (ROMERO, 2010 apud LORENZINI; CEPPI, 2013).
} 
Brasil. Chávez reservaba las propuestas más radicales para el ALBA, pero no exigía la adhesión de Brasil a estos proyectos. Por el contrario, pidió unirse al MERCOSUR, y aceptó acuerdos más pragmáticos para viabilizar a UNASUR y el Banco del Sur (ALVES DO CARMO, 2012).

En el caso de los liderazgos regionales sudamericanos, entonces, Brasil lo ha ejercido a través del poder blando (MALAMUD, 2011), pero siempre utilizando la persuasión y la creación de consenso para perseguir intereses regionales. En cuanto a Venezuela, su liderazgo regional se ha ejercido de una manera más ideológica y reaccionaria, oponiéndose al libre mercado, y a través de la "diplomacia petrolera" (ALTMANN BORBÓN, 2009 apud QUILICONI; RIVERA, 2019, p.227).

Los liderazgos de estos dos países se han ido desvaneciendo, sin embargo, entre una Venezuela sumida en la crisis económica, política y social, y amenazada por el decreciente rol de su economía petrolera - aunque manteniendo su discurso y su proyección ideológicos -, y un Brasil que ha virado a la derecha y que busca abrirse, luego de fuertes vaivenes en su coyuntura doméstica a una interlocución creciente (aunque no sin contradicciones) con China, con Estados Unidos y con la Unión Europea, en una perspectiva pragmática.

\section{La convergencia ideológica en el ocaso del regionalismo post-liberal}

Una década después del surgimiento de esquemas subregionales de cooperación e integración con tono progresista como ALBA y UNASUR, asistimos a fuertes movimientos en detrimento de su supervivencia. Especialmente, una nueva concepción de intereses comunes suscitada en gobiernos conservadores de varios países sudamericanos predispuso a la creciente fragilidad de los esquemas post-liberales. A medida que el signo político de los gobiernos de los países sudamericanos fue girando a la derecha, lo hicieron también sus intereses en política exterior. ALBA y UNASUR dejaron de ser importantes para las agendas exteriores de los países de la región.

UNASUR, para muchos, se transformó en una forma de mantener el legado de una izquierda que había sido derrotada en las urnas y, en el caso de Venezuela, había iniciado un camino de ruina. Un claro proceso de despolitización dio comienzo, especialmente en términos institucionales, a partir de enero de 2017, cuando la organización quedó acéfala y su funcionamiento prácticamente se redujo a lo elemental, al menos en contraste con el protagonismo 
político que tuvo desde su fundación hasta $2016 .{ }^{14}$ Los disensos en torno a la crisis en Venezuela, y la declinación de las prioridades de los gobiernos respecto del ordenamiento regional desgastaron su mandato y capacidad de respuesta a las tensiones y coyunturas regionales.

En abril de 2018, los gobiernos de Argentina, Brasil, Chile, Colombia, Paraguay y Perú decidieron suspender temporalmente su membresía. Esa decisión se tomó en "un contexto de creciente activismo político-diplomático de estos países desde el Grupo de Lima” para la solución de la crisis en Venezuela (SANAHUJA, 2019, p.114). Posteriormente, a lo largo del año 2019, esos gobiernos, junto con el de Ecuador, formalizaron su salida del bloque. Más aún, luego de la renuncia de Evo Morales en Bolivia, el gobierno interino tiene la intención de desvincularse también. En palabras de su canciller Karen Longaric, "En los hechos (Unasur) no existe, no opera y no nos beneficia para nada" (BOLIVIA..., 2019a).

Hoy, el colapso de la UNASUR, impensable hace diez años en muchos círculos que promovieron la institucionalización del organismo, parece inevitable. Sin embargo, y mientras siga presente una mayoría de gobiernos conservadores, el "vaciamiento" de UNASUR intenta ser disimulado con la creación de PROSUR. La convergencia de signo ideológico entre gobiernos sudamericanos dejaba de producirse en clave progresista, y se movía hacia la derecha. Este nuevo esquema, creado en marzo de 2019 por ocho países sudamericanos, se erige en torno a intereses comunes en pos de objetivos políticos concretos: aislar a Venezuela y hacer desaparecer a la UNASUR. ${ }^{15}$

Pero la creación de PROSUR, un esquema supuestamente "desideologizado", está, contrariamente, repleto de razones ideológicas. Los gobiernos firmantes de la Declaración de Santiago comparten el interés de que se deben realizar elecciones libres, transparentes y con observadores internacionales en Venezuela, siendo que estos países consideran que el gobierno de Nicolás Maduro es ilegal e ilegítimo, y debilitar a UNASUR, organismo paradigmático de los gobiernos progresistas de Lula, Kirchner y Chávez. El propio Bolsonaro expresó que PROSUR era un nuevo espacio de diálogo e integración en Sudamérica, opuesto al "avance totalitario" observado en el continente en los últimos ańos con UNASUR (ROSA, 2019). Más allá de estas metas, no queda claro qué tipo

\footnotetext{
${ }^{14}$ Incluso el Parlamento de UNASUR nunca se creó. Paradójicamente, sin contar siquiera con un instrumento jurídico de creación, se construyó el edificio que albergaría su sede en Cochabamba, Bolivia. El establecimiento de este parlamento regional parecía más un negocio para las empresas constructoras y el gobierno boliviano que un paso hacia la democratización del proceso (ALVAREZ, 2017).

${ }^{15}$ Los ocho países firmantes de la Declaración de Santiago son: Argentina, Brasil, Chile, Colombia, Ecuador, Perú, Paraguay y Guyana.
} 
de cooperación o integración se quiere llevar adelante. Como sostiene Frenkel (2019), reemplazar un organismo plural e inclusivo por otro limitado a gobiernos afines impide desarrollar mecanismos efectivos de resolución de conflictos y espacios plurales desde los cuales consensuar mínimos denominadores comunes.

La ideología también ha cedido en el caso del ALBA. Desde la muerte de Chávez, en marzo de 2013, el bloque mostraba una agenda menos activa y no se vislumbraba una coincidencia evidente en torno a los intereses comunes que organizaron al esquema. Más aún, para todos los Estados miembros - menos Cuba -, ALBA era sólo una de las diversas formas de integración regional que se perseguían en paralelo, y "no necesariamente la que se priorizaba" (CUSACK, 2019, p.107).

ALBA fue perdiendo miembros a medida que sus gobiernos giraban hacia la derecha. Primero fue el gobierno ecuatoriano, que anunció su retiro diez años después de su ingreso, por el manejo venezolano de la crisis migratoria. ${ }^{16}$ El gobierno de Lenín Moreno - quien fue vicepresidente de Correa - se fue distanciando de la anterior administración y emprendiendo importantes virajes a pesar de sus orígenes progresistas, especialmente en política económica y política exterior.

Más recientemente ha sido el gobierno interino de Bolivia, presidido por Jeanine Áñez, el que decidió abandonar el bloque. El gobierno interino rompió relaciones con el gobierno venezolano y anunció su retiro del ALBA. Pero, a su vez, ALBA no reconoció al nuevo gobierno en Bolivia. Un día antes del anuncio del gobierno boliviano, el Consejo Político del ALBA sesionó en Nicaragua y condenó lo que definió como "golpe de Estado consumado" contra Morales "de la oligarquía boliviana” (BOLIVIA..., 2019b).

En la actualidad, las reuniones de algunos de sus consejos - especialmente el Consejo Político- y la celebración de reuniones cumbres entre Venezuela y sus (cada vez menos) aliados ideológicos como Cuba y Nicaragua ${ }^{17}$ no logran disimular cierto retroceso del bloque en términos concretos de integración y cooperación, que intenta ser compensada con una retórica ideologizada cada vez más exaltada. ${ }^{18}$

\footnotetext{
${ }^{16}$ Asimismo, el gobierno de Lenín Moreno anunció el fin del convenio bilateral con Cuba por el que prestaban servicios alrededor de 400 médicos cubanos destinados en Ecuador (ECUADOR..., 2018).

${ }^{17}$ El gobierno nicaragüense de Ortega está fuertemente cuestionado por una crisis político - institucional iniciada en 2018. La situación ha dejado al menos 320 muertos, centenares de prisioneros, miles de exiliados y millonarias pérdidas económicas (WALLACE, 2019).

${ }^{18}$ El Salvador fue aliado de Venezuela hasta que asumió el gobierno Nayib Bukele, quien reconoció a Juan Guaidó como presidente encargado de Venezuela. En noviembre de 2019, el conflicto llegó al punto en que ambos países expulsaron a sus respectivos cuerpos diplomáticos (EL SALVADOR..., 2019).
} 
ALBA nunca obtuvo gran influencia política o económica, ni dentro ni fuera de Sudamérica y el Caribe. Al proyecto de ALBA sólo se unieron pequeños países pobres con mínima influencia internacional. Inclusive, hasta se podría cuestionar la afinidad ideológica entre sus miembros: la mayoría de los demás países fueron más moderados en su antiamericanismo o simplemente apoyaron plenamente a Estados Unidos (CORRALES; ROMERO, 2015).

En realidad, resulta difícil no relativizar la vocación post-hegemónica del ALBA mientras uno de sus países es parte del DR-CAFTA, otro tenía el dólar como moneda oficial y el socio comercial más importante del líder del grupo es Estados Unidos (BRICEÑO RUIZ, 2014). Incluso Ecuador, que había ingresado al ALBA con el gobierno de Correa, firmó en 2016, con ese mismo gobierno, el Tratado multipartes con la Unión Europea (uniéndose a Colombia y Perú), y, finalmente, se retiró del ALBA en 2018. Bolivia, por su parte, no solo nunca abandonó la CAN, sino que solicitó ser miembro pleno de MERCOSUR (al igual que la propia Venezuela).

\section{El liderazgo regional en el ocaso del regionalismo post-liberal}

El liderazgo de dos potencias medias como Brasil y Venezuela - gobernados por presidentes con vocación de liderazgo - explican, en parte, la aparición de estructuras regionales como ALBA y UNASUR. Pero el declive de las potencias regionales que pergeñaron la gobernanza regional en clave post-liberal está conectado con el debilitamiento de ese modelo de gobernanza.

En los últimos años, Brasil se ha visto inmerso en severas crisis políticas e institucionales. Los factores de carácter doméstico son los que aparecen identificados como los de mayor relevancia en la degradación del liderazgo sobre la región sudamericana de este país (CAETANO; LÓPEZ BURIAN; LUJÁN, 2019). El compromiso de Brasil como líder regional comenzó a decaer durante el gobierno de Dilma Rousseff (2011-2016), no porque no haya sido controvertido con anterioridad, sino porque aumentaron las dudas sobre la relevancia de las estructuras regionales para el país. A su vez, la presidencia de Rousseff coincidió con un empeoramiento de la situación económica, una mala gestión política, y una política exterior más retraída.

Esa combinación de crisis interna y menor proyección externa significó un claro declive de Brasil como actor regional e internacional. Ello se profundizó aún más con el proceso de impeachment de Rousseff y la subsecuente asunción de Michel Temer, eventos que terminaron con escasas credenciales democráticas un periodo que había establecido como prioridad política la construcción 
de un liderazgo en la región (GOMES SARAIVA, 2010). Actualmente, el liderazgo regional no constituye una prioridad para el gobierno de Bolsonaro: su política exterior se estructura en términos antitéticos respecto de los gobiernos petistas. Se evita estrechar relaciones con los países vecinos, en pos de la "desamericanización", que propone un distanciamiento de Brasil de la región (FRENKEL, 2018).

Si el gobierno de Temer suspendía la membresía de Brasil en UNASUR en el momento en que lo hacían los gobiernos de Macri en Argentina, de Piñera en Chile, y de Duque en Colombia -, el de Bolsonaro consumó la desvinculación del bloque y acompañó entusiastamente la iniciativa chilena-colombiana de PROSUR. Ninguno de los países que impulsaron esta iniciativa, sin embargo, puede liderarla. Chile y Colombia están ensimismados en la resolución de graves problemas internos, Brasil no tiene afán ni deseo alguno en liderar este foro ni ningún otro en la región, y Argentina ha virado nuevamente a la centroizquierda con el gobierno de Alberto Fernández. Las debilidades en unos y el desinterés en otros conducen al PROSUR a un final anticipado antes de que haya levantado vuelo.

PROSUR no tiene las condiciones para consolidarse como un bloque regional sudamericano. $\mathrm{Y}$ es probable que sus líderes, alineados con el discurso de la integración flexible, tampoco quieran que sea un acuerdo a largo plazo. Tal vez sea posible verlo como una plataforma para enviar un mensaje a Venezuela. Desmantelando UNASUR se aisló al gobierno de Nicolás Maduro, erigiéndose como una herramienta adicional de presión contra el régimen.

En tanto, Venezuela se encuentra inmersa en una espiral negativa de crisis política, económica y social sin precedentes. Utilizando nuestro marco conceptual, resulta evidente que la dimensión que más se ha resentido en los últimos años en el liderazgo venezolano ha sido la vinculada con los recursos, no con los recursos discursivos o ideológicos sino con aquellos más tangibles vinculados a la renta petrolera y su potencialidad para cohesionar voluntades en torno de un proyecto en común.

Hacia 2012, en pleno apogeo del ALBA, ya existían dudas sobre las capacidades de Venezuela para construir los consensos que requería un proyecto de integración viable, debido a su marcada orientación ideológica. Además, se reconocía que era difícil de sostener a partir del voluntarismo político de alianzas y de los recursos que Venezuela desplegaba en apoyo de ese proyecto, en particular, la posibilidad de extender el modelo rentista petrolero al conjunto de la región (SERBIN, 2011; SANAHUJA, 2012). 
Algunos han señalado que ALBA se asemejaba a un mero proyecto propagandístico cuyo futuro y capacidad de recuperación estaba condicionado a la presencia de Chávez y más significativamente a los ingresos del petróleo. En realidad, los más optimistas se equivocaron al afirmar que, a diferencia de otros proyectos regionales en América, la importancia del ALBA y su resiliencia estaban dadas por el impacto de los proyectos y redes regionales de solidaridad, “incluso más allá de Chávez” (RIGGIROZZI, 2012b, p.435).

Especialmente desde la asunción de Maduro, no se vislumbra un liderazgo evidente en torno a los principios que organizaron al mecanismo. El modelo funcionó durante un tiempo, pero tenía una debilidad: su dependencia financiera de Venezuela. Este país era el paymaster que permitía que el modelo del ALBA haya funcionado durante unos años y, en Petrocaribe, era quien entregaba el petróleo en condiciones especiales (BRICEÑO RUIZ, 2014). Era claro que la política estratégica de alianzas dependía de los vaivenes económicos de la Venezuela post-chavista. El descalabro de la economía venezolana ha sido determinante en este relativo fracaso, porque acota las posibilidades de usar el petróleo en las acciones de política exterior.

No solo el ALBA ha perdido miembros sino que, además, comparado con sus ambiciosos objetivos, los resultados del esquema son modestos. Entre ellos: el canal mediático Telesur, Petrocaribe, Misión Milagros y las Casas Culturales ALBA. Las demás iniciativas, incluyendo el Banco ALBA, el Sistema Unitario de Compensación Regional de Pagos (SUCRE), cadenas complementarias de producción o una mayor autonomía alimentaria, están estancadas o no se han realizado (GRATIUS; PUENTE, 2018, p.242).

Guatemala se retiró de Petrocaribe en 2014 y Belice en 2017, debido a la brusca caída de la producción petrolera venezolana, que no permitía respetar los acuerdos. Finalmente, en junio de 2018, Venezuela anunció la suspensión de sus envíos a Antigua y Barbuda, Dominica, El Salvador, Nicaragua, San Vicente y las Granadinas, San Cristóbal y Nieves y Haití. En cuanto a los resultados de esta iniciativa, no se han visto mejoras significativas en las sociedades y en muchos casos ha financiado la corrupción gubernamental (ANTONIN, 2018), ${ }^{19}$ como trágicamente se manifestó en Haití, donde las protestas contra el gobierno por corrupción y malversación de fondos de Petrocaribe dejaron numerosos muertos.

\footnotetext{
19 De todas maneras, es difícil conocer en qué estado se encuentran realmente estos procesos ya que la ausencia de un sistema de monitoreo y la escasa presencia de actores de la sociedad civil no permiten un seguimiento regular de sus actividades y decisiones (GRATIUS; PUENTE, 2018).
} 
Cuando los precios del petróleo se desplomaron en 2014, los efectos sobre la ya deteriorada economía venezolana fueron catastróficos, con una inflación desenfrenada y la escasez de alimentos y medicamentos. El fracaso público del socialismo del siglo XXI no sólo extinguió el antiguo faro de ideas postliberales de Venezuela, sino que también limitó severamente la capacidad del país para actuar como paymaster de los proyectos regionales, ya que la financiación discrecional ha sido redirigida hacia el ámbito nacional (CUSACK, 2019).

\section{Conclusiones}

En este trabajo nos propusimos analizar de qué manera elementos como la convergencia ideológica entre los ejecutivos nacionales y el liderazgo regional de un país asumen cierta capacidad explicativa a la hora de dilucidar los patrones y vaivenes de los esquemas regionales en América Latina.

A la hora de dar cuenta de la conformación de un bloque regional o explicar el logro de consensos a nivel regional, la afinidad política entre gobiernos es una variable para considerar en el análisis. A medida que el signo político de las presidencias de los países sudamericanos fue girando a la derecha, lo hicieron también las agendas de política exterior. En esas agendas, UNASUR y ALBA dejaron de ser importantes. Para muchos, esos esquemas se transformaron en una forma de mantener el legado de una izquierda que, en el mejor de los casos, había sido derrotada en las urnas y, en el peor, había iniciado un camino de colapso y derrumbe, como en Venezuela.

Parece indiscutible que, al haber sido capaz de impulsar instituciones como UNASUR, Brasil desempeñó un papel importante en la promoción de la gobernanza regional, respondiendo en buena medida a su habilidad para integrar dentro de la misma organización países con políticas económicas y exteriores diferentes. Sin embargo, en un contexto menos halagüeño que el de la década anterior, el gobierno de Rousseff tuvo dificultades para mantener los logros y las responsabilidades del liderazgo brasileño. Estos inconvenientes, sumados a la destitución de Dilma y la llegada de un gobierno liberal-conservador, modificaron las prioridades en política exterior y debilitaron las pretensiones de liderazgo. El gobierno de Bolsonaro, a su vez, ha ratificado el rumbo de una política exterior alejada de preferencias y dinámicas regionales.

UNASUR se desmanteló a partir de las decisiones de más de la mitad de los gobiernos de abandonar el bloque, como hemos comentado. Durante su época de oro, fue la divergencia de preferencias lo que caracterizó a la organización, y, a su vez, fue el liderazgo brasileño el factor aglutinador. El 
debilitamiento del esquema estuvo más vinculado a la falta de interés por parte de Brasil que a cualquier otro elemento, especialmente frente a la divergencia de posiciones generadas por la crisis en Venezuela.

En el caso de ALBA, el declive del líder significó el inicio de su decadencia. Consecuentemente con el descalabro político, económico y social de Venezuela, y un descenso abrupto en la renta petrolera de ese país, el esquema se debilitó. Es cierto que hay una gran cantidad de discurso ideológico y simbolismo en la conformación del ALBA. Pero el esquema era contingente al ciclo político y al voluntarismo político que comportaba el liderazgo de Venezuela, a su vez condicionado por un modelo "rentista" que la caída de los precios del petróleo hace inviable (SERBIN, 2011). Ese es el factor fundamental a la hora de explicar el debilitamiento del bloque. Los cambios en la correlación de fuerzas domésticas y la decisión de abandonar el esquema por parte de gobiernos recientes en Ecuador y Bolivia, en realidad, son posteriores a la crisis de liderazgo de Venezuela.

La Venezuela de Hugo Chávez tenía como objetivo formular y presentar un nuevo modelo de integración regional, una "integración alternativa", para los países sudamericanos y latinoamericanos en general. La propuesta de ALBA habría sido un paso en ese sentido, pero hoy parece claro que, entre la expectativa inicial y la realidad, ese nuevo paradigma de integración no fue alcanzado (ALVES DO CARMO, 2012).

En definitiva, las propuestas post-liberales pierden dinamismo y sustento una vez que se debilitan los liderazgos regionales que las impulsaron de la mano de la sustitución de presidentes como Lula o Chávez por mandatarios con menos vocación y/o posibilidades de liderar, y no simplemente cuando la confluencia de ideas e intereses desaparece. En este sentido, parecería manifestarse en la región un hecho fundamental: en los últimos años la decadencia del poder de Brasil y Venezuela contribuye a dar cuenta de los cambios y de las reversiones políticas en la gobernanza regional, tal como lo demuestran instancias como UNASUR y ALBA.

Subsisten aún numerosos interrogantes a ser respondidos. Resulta crucial que futuras investigaciones - que involucren una mayor cantidad de variables en el análisis - puedan ser emprendidas. Hay espacio suficiente para realizar avances en la interpretación de las fluctuantes fases del regionalismo latinoamericano en general, y sudamericano, en particular. Para desarrollar un panorama completo de este fenómeno, se necesitarán estudios adicionales que expliquen un proceso aún en movimiento. 


\section{REFERENCIAS}

ALVAREZ, M. V. La declinación del liderazgo y el regionalismo: los casos de Brasil en UNASUR y Venezuela en ALBA. En: LECHINI, G.; MORASSO, C. (comp.). Auge y ocaso de los emergentes en el siglo XXI: ¿Cambios sistémicos, domésticos o cosméticos?. Rosario: UNR Editora, 2020. p. 30-54.

ALVAREZ, M.V. La representación política en espacios transnacionales: una comparación de los parlamentos regionales en América Latina, Cuadernos del CIESAL, Rosario, Argentina, v.14, n.16, p. 49-76, 2017.

ALVES DO CARMO, C. O projeto integracionista bolivariano de Hugo Chávez e o pragmatismo da política externa brasileira. En: SERBIN, A.; MARTÍNEZ, L.; RAMANZINI JÚNIOR, H. (coord.). Anuario de la Integración Regional de América Latina y el Gran Caribe 2012. Buenos Aires: CRIES, 2012. p. 301-320.

ANTONIN, A. ¿Dónde está el dinero de Petrocaribe? Las protestas anticorrupción que acorralan al gobierno haitiano, Nueva Sociedad, Buenos Aires, diciembre 2018. Disponible en: http://nuso.org/articulo/haiti-corrupcion-petrocaribe-venezuela/. Acceso en: 12 ene. 2021.

BOLIVIA deja la ALBA-TCP y estudia su salida de la Unasur. La Razón, 15 nov. 2019a. Disponible en: https://www.la-razon.com/nacional/2019/11/15/bolivia-dejala-alba-tcp-y-estudia-su-salida-de-la-unasur/. Acceso en: 13 ene. 2021.

BOLIVIA rompe relaciones con Venezuela y se retira de la Alianza Bolivariana ALBA. Deutsche Welle, Bonn, Alemania, 15 nov. 2019b. Disponible en: https:// www.dw.com/es/bolivia-rompe-relaciones-con-venezuela-y-se-retira-de-la-alianzabolivariana-alba/a-51271927. Acceso en: 15 ene. 2021.

BRICEÑO RUIZ, J. El ALBA: una discusión de su modelo, sus resultados y sus perspectivas. En: SERBIN, A.; MARTÍNEZ, L.; RAMANZINI JÚNIOR, H. (coord.). Anuario de la Integración Regional de América Latina y el Caribe. Buenos Aires: CRIES, 2014. p. 151-178.

BRICEÑO RUÍZ, J. La Alianza del Pacífico: la viabilidad de un naciente bloque regional. En: ARDILA, M. (ed.). El Pacífico latinoamericano y su inserción internacional. Bogotá: Pontificia Universidad Javeriana, 2012. p. 135-158.

BURGES, S. W. Brazilian Foreign Policy after the Cold War, Gainesville: University Press of Florida, 2009.

CAETANO, G.; LÓPEZ BURIAN, C.; LUJÁN, C. Liderazgos y regionalismos en las relaciones internacionales latinoamericanas, Revista CIDOB d'Afers Internacionals, Barcelona, n.121, p. 181-207, 2019. 
COMINI, N.; FRENKEL, A. Una Unasur de baja intensidad: Modelos en pugna y desaceleración del proceso de integración en América del Sur, Nueva Sociedad, Buenos Aires, n.250, p. 58-77, marzo-abril 2014.

CORRALES, J.; ROMERO, C. A. Venezuela's foreign policy, 1920s-2010s. En: DOMÍNGUEZ, J. I.; COVARRUBIAS, A. (ed.). Routledge Handbook of Latin America in the World. Londres: Routledge, 2015. p. 153-168.

CUSACK, A. K. Venezuela, ALBA, and the Limits of Post-neoliberal Regionalism in Latin America and the Caribbean. Nueva York: Palgrave Macmillan, 2019.

DABÈNE, O. The politics of regional integration in Latin America: Theoretical and Comparative Explorations. Basingstoke: Palgrave Macmillan, 2009.

DA MOTTA, P.; RÍOS, S. O Regionalismo pós-liberal na América do Sul: origens, iniciativas e dilemas. Santiago de Chile: CEPAL, 2007. (Serie Comercio Internacional, 62).

ECUADOR anuncia su salida del ALBA para presionar a Venezuela por el éxodo masivo de sus ciudadanos. El País, Madrid, 24 ag. 2018. Disponible en: https://elpais. com/internacional/2018/08/24/america/1535066311_810891.html. Acceso en: 5 ene. 2021.

EL SALVADOR y Venezuela expulsan a sus respectivos cuerpos diplomáticos. Telam, Buenos Aires, 3 nov. 2019. Disponible en: https://www.telam.com.ar/ notas/201911/405474-el-salvador-y-venezuela-expulsan-a-sus-respectivos-cuerposdiplomaticos.html. Acceso en: 10 ene. 2021.

FLEMES, D.; WOJCZEWSKI, T. Contested leadership in international relations: power politics in South America, South Asia and Sub-Saharan Africa. GIGA Working Papers, Hamburg, Germany, n.121, p. 1-35, 2010.

FRENKEL, A. PROSUR: el último Frankenstein de la integración sudamericana. Nueva Sociedad, Buenos Aires, jun. 2019. Disponible en: https://nuso.org/articulo/ prosur-integracion-america-latina-derecha-alianza/. Acceso en: 5 ene. 2021.

FRENKEL, A. El mundo según Bolsonaro: La nueva política exterior de Brasil. Nueva Sociedad, Buenos Aires, nov. 2018. Disponible en: https://nuso.org/articulo/ el-mundo-segun-bolsonaro/. Acceso en: 5 ene. 2021.

GILPIN, R. U.S. Power and the Multinational Corporation. Nueva York: Basic Books, 1975.

GOMES SARAIVA, M. Procesos de integración de América del Sur y el papel de Brasil: los casos del MERCOSUR y la UNASUR. Revista CIDOB d'afers internacionals, Barcelona, n.97-98, p. 87-100, 2012. 
GOMES SARAIVA, M. Brazilian foreign policy towards South America during the Lula Administration: caught between South America and MERCOSUR. Revista Brasileira de Política Internacional, Brasília, v.53, ed. esp., p. 151-168, 2010.

GRATIUS, S.; PUENTE, J. M. ¿¿Fin del proyecto alternativo ALBA?: Una perspectiva política y económica, Revista de Estudios Políticos, Madrid, n.180, p. 229-252, 2018.

HAAS, E. B. The Uniting of Europe: Political, Social, and Economic Forces, 1950-1957. 2. ed. Stanford, CA: Stanford University Press, 1968.

HAAS, E. B. The Uniting of Europe: Political, Social, and Economic Forces: 1950-1957. Stanford, CA: Stanford University Press, 1958.

HERRERA SANTANA, D. Hegemonía y Relaciones Internacionales: Un estado del arte. Revista de Relaciones Internacionales de la UNAM, Ciudad de Mexico, n.127, p. 11-47, 2017.

KNORR, K. The Power of Nations: The Political Economy of International Relations. Nueva York: Basic Books, 1975.

KRASNER, S. D. State Power and the Structure of International Trade. World Politics, New York, v.28, n.3, p. 317-347, 1976.

LEGLER, T. Post-hegemonic regionalism and sovereignty in Latin America: optimists, skeptics, and an emerging research agenda. Contexto Internacional, Rio de Janeiro, v.35, n.2, p. 325-352, 2013.

LÓPEZ CANELLAS, M. F.; VILANI, D. El acuerdo Petrocaribe en el marco de la Cooperación Sur: Sur y su relevancia política y económica. En: SERBIN, A.; MARTÍNEZ, L.; RAMANZINI JÚNIOR, H. (coord.). Anuario de la Integración Regional de América Latina y el Caribe. Buenos Aires: CRIES, 2014. p. 179-206.

LORENZINI, M.E.; CEPPI, N. Bolivia y Venezuela como PRM's: Un análisis comparativo en torno a la cooperación internacional. En: COLACRAI, M. (comp.). La Cooperación Internacional desde la visión de los PRM: discusiones conceptuales, diseños de políticas y prácticas sudamericanas. Rosario: UNR Editora, 2013. p. 189-224.

MALAMUD, A. Presidentialist decision making in Latin American foreign policy. Examples from Regional Integration Processes. En: DOMINGUEZ J.I.; COVARRUBIAS, A. (ed.). Routledge Handbook of Latin America in the World. Londres: Routledge, 2015. p. 112-123. 
MALAMUD, A. A Leader Without Followers?: The Growing Divergence Between the Regional and Global Performance of Brazilian Foreign Policy. Latin American Politics and Society, Cambridge, UK, v.53, n.3, p. 1-24, 2011.

MALAMUD, A. Conceptos, teorías y debates sobre la integración regional. En: CONGRESO LATINOAMERICANO DE CIENCIA POLÍTICA (ALACIP), 5., Buenos Aires. Anales [...], Buenos Aires, 28-30 jul. 2010. p.1-23.

MALAMUD, A.; GARDINI, G. L. Has Regionalism Peaked?: The Latin American Quagmire and its Lessons. The International Spectator: Italian Journal of International Affairs, Roma, v.47, n.1, p. 116-133, 2012.

MATTLI, W. The logic of Regional Integration: Europe and Beyond. Cambridge: Cambridge University Press, 1999.

MORAVCSIK, A. The Choice for Europe: Social Purpose and State Power from Messina to Maastricht. Ithaca, NY: Cornell University Press, 1998.

MOURÓN, F.; ONUKI, J. ¿¿Brasil es un líder en América del Sur?: El papel brasileiro através del concepto de liderazgo situacional. Estudos Internacionais, Belo Horizonte, v.3, n.1, p. 9-25, 2015.

NOLTE, D. Potencias regionales en la política internacional: conceptos y enfoques de análisis. GIGA Working Papers, Hamburg, Germany, n.30, 2006. Disponible en: https://www.researchgate.net/publication/5080737_Potencias_regionales_en_la_politica_internacional_conceptos_y_enfoques_de_analisis. Acceso en: 5 ene. 2021.

PETERSEN, M.; SCHULZ, C.-A. Setting the Regional Agenda: A Critique of Posthegemonic Regionalism. Latin American Politics and Society, Cambridge, UK, v.60, n.1, p.102-127, 2018.

QUILICONI, C.; RIVERA, R. Ideología y liderazgo en la cooperación regional: los casos del Consejo Suramericano de Defensa y el Consejo Suramericano sobre el problema mundial de las Drogas en UNASUR. Revista Uruguaya de Ciencia Política, Montevideo, Uruguay, v.28, n.1, p. 219-248, 2019.

QUILICONI, C.; SALGADO ESPINOZA, R. Latin American Integration: Regionalism à la Carte in a Multipolar World?. Colombia Internacional, Bogota, v.92, p. 15-41, 2017.

RABY, D. Venezuelan Foreign Policy under Chávez, 1999-2010: The Pragmatic Success of Revolutionary Ideology?. En: GARDINI, G. L.; LAMBERT, P. Latin American Foreign Policies Between Ideology and Pragmatism. Basingstoke: Palgrave Macmillan, 2011. p. 159-177. 
RIGGIROZZI, P. Re-territorializando consensos: Hacia un regionalismo posthegemónico en América Latina. En: SERBIN, A.; MARTÍNEZ, L.; RAMANZINI JÚNIOR, H. (coord.). Anuario de la Integración Regional de América Latina y el Gran Caribe 2012. Buenos Aires: CRIES, 2012a. p. 129-151.

RIGGIROZZI, P. Region, Regionness and Regionalism in Latin America: Towards a New Synthesis, New Political Economy, London, v.17, n.4, p. 421443, $2012 \mathrm{~b}$.

RIGGIROZZI, P.; TUSSIE, D. The Rise of Post-hegemonic Regionalism: The Case of Latin America. London: Springer, 2012.

RIVAROLA PUNTIGLIANO, A.; BRICEÑO-RUIZ, J. Resilience of Regionalism in Latin America and the Caribbean. Nueva York: Palgrave Macmillan, 2013.

ROSA, A. B. Prosul: Entenda como deve funcionar novo bloco de países sulamericanos. Huffpost Brasil, 23 mar. 2019.

SANAHUJA, J.A. La crisis de integración y el regionalismo en América Latina: giro liberal-conservador y contestación normativa. En: MESA, M. (coord.). Ascenso del nacionalismo y el autoritarismo en el sistema internacional Anuario 2018-2019. Madrid: CEIPAZ, 2019. p. 107-126.

SANAHUJA, J.A. Regionalismo e integración en América Latina: de la fractura Atlántico-Pacífico a los retos de una globalización en crisis. Pensamiento Propio, Madrid, v.44, n.21, p. 29-75, 2016.

SANAHUJA, J.A. Regionalismo post-liberal y multilateralismo en Sudamérica: El caso de UNASUR. En: SERBIN, A.; MARTÍNEZ, L.; RAMANZINI JÚNIOR, H. (coord.). Anuario de la Integración Regional de América Latina y el Gran Caribe 2012. Buenos Aires: CRIES, 2012. p. 19-71.

SANAHUJA, J.A. La construcción de una región: Suramérica y el regionalismo posliberal. En: CIENFUEGOS, M.; SANAHUJA, J. A. Una región en construcción: UNASUR y la Integración de América del Sur. Barcelona: CIDOB, 2010. p. 87-134.

SERBIN, A. Déficit democrático y participación ciudadana en el marco del regionalismo post-liberal. En: SERBIN, A.; MARTÍNEZ, L.; RAMANZINI JÚNIOR, H. (coord.). Anuario de la Integración Regional de América Latina y el Gran Caribe 2012. Buenos Aires: CRIES, 2012. p. 73-127. 
SERBIN, A. Regionalismo y soberanía nacional en América Latina: los nuevos desafíos. En: ROJAS ARAVENA, F. (ed.). América Latina y el Caribe: multilateralismo vs. Soberanía. La construcción de la Comunidad de Estados Latinoamericanos y Caribeños. Buenos Aires: Teseo/FLACSO, 2011. p. 49-98.

WALLACE, A. Crisis en Nicaragua, a un año del inicio de las protestas: 'Ortega solo va a ceder cuando la presión nacional e internacional lo pongan en una situación extrema'. BBC Mundo. 18 abr. 2019. Disponible en: https://www. bbc.com/mundo/noticias-america-latina-47964275 Acceso en: 20 nov. 2019.

Recebido em: 25 de agosto de 2020

Aprovado em: 03 de outubro de 2020 